\title{
Nested Stochastic Valuation of Large Variable Annuity Portfolios: Monte Carlo Simulation and Synthetic Datasets
}

\author{
Guojun Gan ${ }^{\mathrm{a}}$, Emiliano A. Valdez ${ }^{\mathrm{a}}$ \\ ${ }^{a}$ Department of Mathematics, University of Connecticut, 341 Mansfield Road U-1009, \\ Storrs, CT, 06269, USA
}

\begin{abstract}
Dynamic hedging has been adopted by many insurance companies to mitigate the financial risks associated with variable annuity guarantees. In order to simulate the performance of dynamic hedging for variable annuity products, insurance companies rely on nested stochastic projections, which is highly computationally intensive and often prohibitive for large variable annuity portfolios. Metamodeling techniques have recently been proposed to address the computational issues. However, it is difficult for researchers to obtain real datasets from insurance companies to test metamodeling techniques and publish the results in academic journals. In this paper, we create synthetic datasets that can be used for the purpose of addressing the computational issues associated with the nested stochastic valuation of large variable annuity portfolios. The runtime used to create these synthetic datasets would be about 3 years if a single CPU were used. These datasets are readily available to researchers and practitioners so that they can focus on testing metamodeling techniques.
\end{abstract}

Keywords: Monte Carlo, Regime-Switching Multivariate Black-Scholes, Metamodeling, Variable Annuity, Portfolio Valuation

Email addresses: Guojun.Gan@uconn.edu (Guojun Gan), emiliano.valdez@uconn.edu (Emiliano A. Valdez) 


\section{Introduction}

A variable annuity (VA) is a popular life insurance product created by insurance companies to address many people's concerns about outliving their assets (Ledlie et al., 2008; The Geneva Association Report, 2013). Under a VA policy, the policyholder agrees to make a lump-sum or a series of purchase payments to the insurer and in return the insurer agrees to make benefit payments to the policyholder, beginning either immediately or on a future date. Policyholders choose to invest their money in one or more investment funds provided by the insurance company. A main feature of VAs is that they come with guarantees or riders, which are designed to protect the policyholder's capital against market downturns.

There are two types of guaranteed benefits embedded in VA policies: death benefits and living benefits. A guaranteed minimum death benefit (GMDB) guarantees a specified amount to the beneficiary upon the death of the policyholder regardless of the performance of the investment portfolio. Examples of living benefits include the guaranteed minimum withdrawal benefit (GMWB), the guaranteed minimum income benefit (GMIB), the guaranteed minimum maturity benefit (GMMB), and the guaranteed minimum accumulation benefit (GMAB). A GMWB guarantees that the policyholder can take systematic annual withdrawals of a specified amount from the policy over a period of time, even though the investment portfolio might be depleted. A GMIB guarantees that the policyholder can convert the VA policy to an annuity according to a specified rate. A GMMB guarantees that the policyholder can receive a specific amount at the maturity of the policy. A GMAB guarantees that the policyholder can renew the contract during a specified window after a specified waiting period.

Due to these attractive guarantees, lots of VA policies were sold in the past two decades. Figure 1 shows the annual VA sales in the United States during the period from 2008 to 2017. From the figure, we see that except for the year 2017, the annual sales in all these years were above $\$ 100$ billion. The guarantees embedded in VA policies are financial guarantees and cannot be adequately addressed by traditional actuarial methods (Boyle and Hardy, 1997). To mitigate the financial risks associated with the VA guarantees, many insurance companies with a VA business have adopted dynamic hedging (Chopra et al., 2009; International Actuarial Association, 2010).

In order to simulate the performance of dynamic hedging for VA products, insurance companies rely on nested stochastic projections (International Ac- 


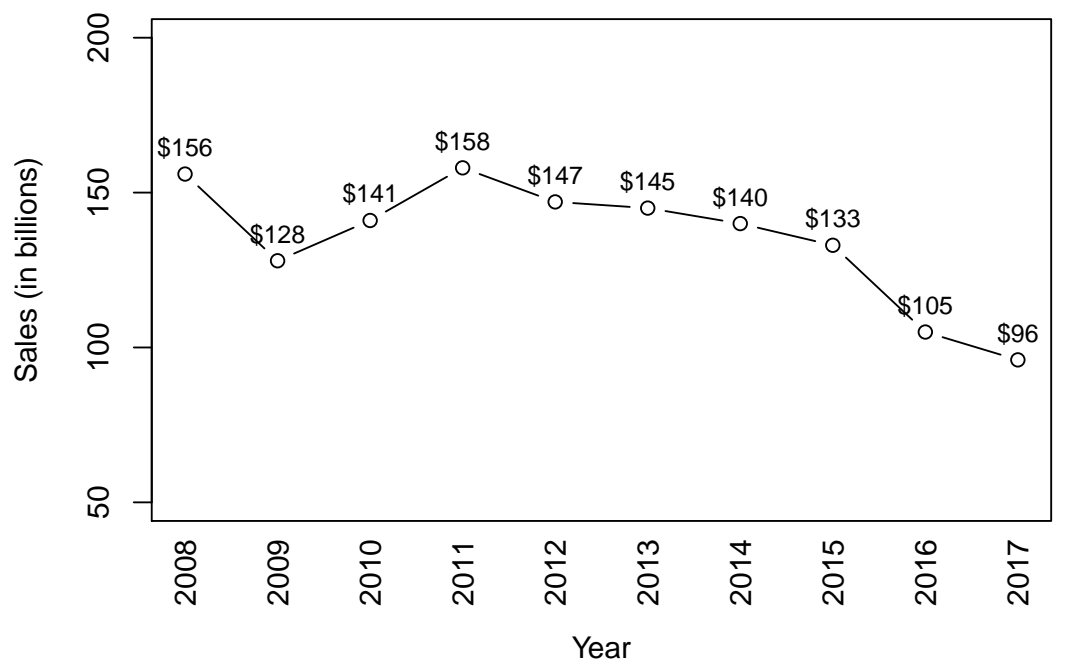

Figure 1: Variable annuity sales in the United States from 2008 to 2017. The numbers are obtained from LIMRA Secure Retirement Institute.

tuarial Association, 2010). Nested stochastic projections are also referred to as "stochastic on stochastic" projections. Figure 2 conceptualizes the structure of a typical nested stochastic projection, which involves two layers of stochastic projections. At each node of an outer stochastic path, a set of inner stochastic paths is embedded. Usually the outer stochastic paths are real-world scenarios, which reflect a realistic pattern of underlying market prices that are used to generate realistic distributions of outcomes. In contrast, the inner stochastic paths are risk-neutral scenarios, which use unrealistic assumptions about risk premiums for purposes of calculating derivative prices under the no-arbitrage assumption.

The computation of nested stochastic projections for a large VA portfolio is highly computationally intensive and often prohibitive because every policy in the portfolio needs to be projected over many paths for a long time horizon (Dardis, 2016). For example, if we use 1000 real-world scenarios in the outer layer and 1000 risk-neutral paths in the inner layer, and project the cash flows at yearly steps for 30 years, then the total number of projections for each policy is

$$
1000 \times 1000 \times 30 \times 31 / 2=4.65 \times 10^{8},
$$

which is already a big number. For a portfolio of 100,000 contracts, the 


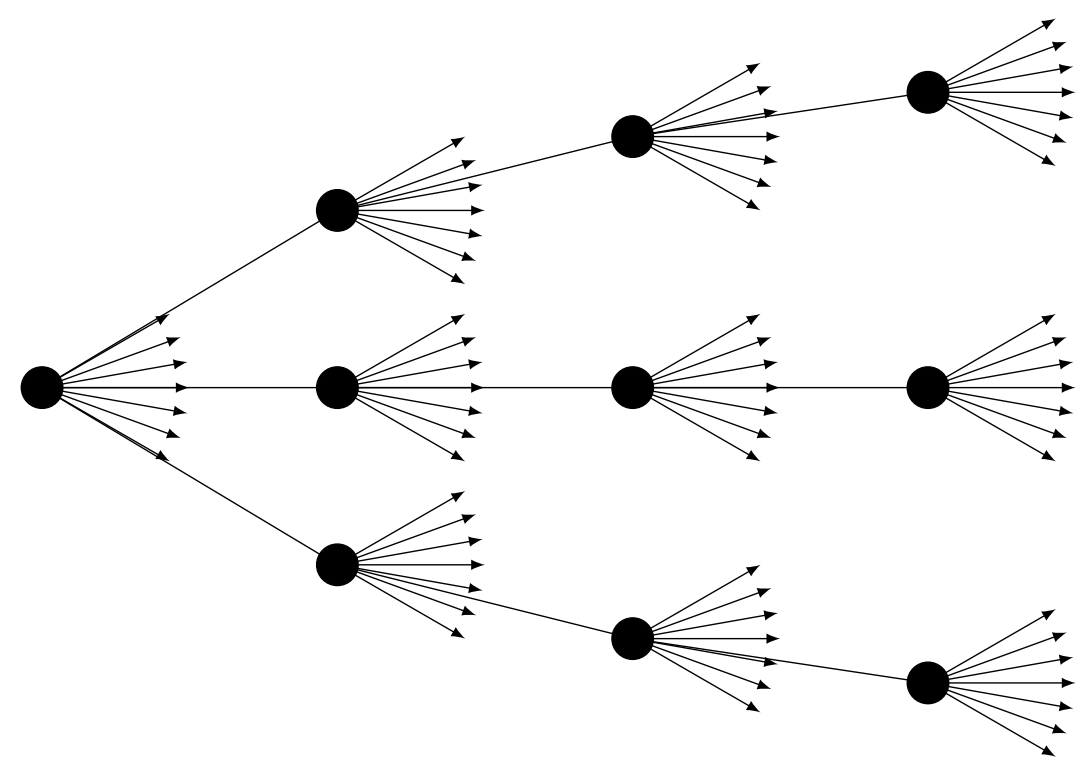

Figure 2: Nested stochastic projections.

number of projections would be $4.65 \times 10^{13}$. Suppose that a single CPU can process 200,000 cash flow projections in a second. Then it will take this CPU

$$
\frac{4.65 \times 10^{13}}{200,000 \times 3600 \times 24 \times 365}=7.37
$$

years to process all the cash flow projections for the portfolio. The amount of time shown in the above equation is just the runtime used to project the cash flows once. To calculate the Greeks, we need to project the cash flows multiple times at different shocks of the market. This will increase the runtime multifold.

Recently, metamodeling approaches have been proposed to address the computational issues associated with the valuation of large VA portfolios. See, for example, Gan (2013), Gan and Lin (2015), Gan (2015), Gan and Lin (2016), Gan and Valdez (2016), Hejazi and Jackson (2016), Gan and Huang (2017), Hejazi et al. (2017), Gan and Valdez (2018), and Xu et al. (2018). The main idea of these metamodeling approaches is to build a predictive model based on a set of representative VA policies and their fair market values (or other quantities of interest). The predictive model is then used to estimate the fair market values for all the policies in the portfolio. This can reduce the number of policies that are valued by Monte Carlo simulation. Since 
predictive models are usually much faster than Monte Carlo simulation, the gain in valuation runtime is significant.

However, it is difficult for academic researchers to obtain real datasets from insurance companies to assess the performance of metamodeling techniques. In this paper, we create synthetic datasets that can be used by researchers and practitioners to test metamodeling methods for the efficient valuation of large VA portfolios under nested stochastic simulation. In particular, we implement a nested stochastic valuation engine that is used to calculate the Greeks for VA policies along outer layer paths. The purpose of this work is to relieve researchers from spending time on creating such datasets, which can be extremely time-consuming to create.

The remaining part of this paper is structured as follows. Section 2 presents a nested stochastic simulation engine for valuing the guarantees embedded in variable annuities. In Section 3 and Section 4, we present synthetic datasets that can be used to test the performance of metamodeling techniques. In Section 5, we conclude the paper with some remarks. The software that implements the nested Monte Carlo simulation engine is described in Appendix A.

\section{Nested Stochastic Valuation}

In this section, we describe the nested stochastic valuation engine. In particular, we introduce the risk-neutral scenario generator, the real-world scenario generator, and the cash flow projections.

\subsection{Risk-Neutral Scenario Generator}

Risk-neutral scenarios are used in the inner loop to calculate the dollar Deltas. We use a multivariate Black-Scholes model introduced by Carmona and Durrelman (2006) to generate risk-neutral scenarios. This model is also described in Gan and Valdez (2017).

Let $S^{(1)}, S^{(2)}, \ldots, S^{(k)}$ be $k$ indices in the financial market. Under the multivariate Black-Scholes model, the risk-neutral dynamics of the $k$ indices are given by (Carmona and Durrelman, 2006):

$$
\frac{\mathrm{d} S_{t}^{(h)}}{S_{t}^{(h)}}=r_{t} \mathrm{~d} t+\sum_{l=1}^{k} \sigma_{h l} \mathrm{~d} B_{t}^{(l)}, \quad S_{0}^{(h)}=1, h=1,2, \ldots, k
$$


or

$$
S_{t}^{(h)}=\exp \left[\left(\int_{0}^{t} r_{s} \mathrm{~d} s-\frac{t}{2} \sum_{l=1}^{k} \sigma_{h l}^{2}\right)+\sum_{l=1}^{k} \sigma_{h l} B_{t}^{(l)}\right], \quad h=1,2, \ldots, k,
$$

where $B_{t}^{(1)}, B_{t}^{(2)}, \ldots, B_{t}^{(k)}$ are independent standard Brownian motions, $r_{t}$ is the short rate of interest, and the matrix $\left(\sigma_{h l}\right)$ is used to capture the correlation among the indices.

Let $t_{0}=0, t_{1}=\Delta, \ldots, t_{m}=m \Delta$ be time steps with equal space $\Delta$ and suppose that the continuous forward rate is constant within each period. For $j=1,2, \ldots, m$, the accumulation factor of the $h$ th index for the period $\left(t_{j-1}, t_{j}\right)$ can be calculated as:

$$
A_{j}^{(h)}=\frac{S_{j \Delta}^{(h)}}{S_{(j-1) \Delta}^{(h)}}=\exp \left[\left(f_{j}-\frac{1}{2} \sum_{l=1}^{k} \sigma_{h l}^{2}\right) \Delta+\sum_{l=1}^{k} \sigma_{h l} \sqrt{\Delta} Z_{j}^{(l)}\right],
$$

where $f_{j}$ is the annualized continuous forward rate for period $\left(t_{j-1}, t_{j}\right)$ and

$$
Z_{j}^{(l)}=\frac{B_{j \Delta}^{(l)}-B_{(j-1) \Delta}^{(l)}}{\sqrt{\Delta}}
$$

By the property of Brownian motion, we know that $Z_{1}^{(l)}, Z_{2}^{(l)}, \ldots, Z_{m}^{(l)}$ are independent random variables with a standard normal distribution.

The continuous return for the period $\left(t_{j-1}, t_{j}\right)$ is calculated as:

$$
R_{j}^{(h)}=\ln A_{j}^{(h)}=\left(f_{j}-\frac{1}{2} \sum_{l=1}^{k} \sigma_{h l}^{2}\right) \Delta+\sum_{l=1}^{k} \sigma_{h l} \sqrt{\Delta} Z_{j}^{(l)} .
$$

The matrix

$$
\sigma=\left(\begin{array}{cccc}
\sigma_{11} & \sigma_{12} & \cdots & \sigma_{1 k} \\
\sigma_{21} & \sigma_{22} & \cdots & \sigma_{2 k} \\
\vdots & \vdots & \ddots & \vdots \\
\sigma_{k 1} & \sigma_{k 2} & \cdots & \sigma_{k k}
\end{array}\right)
$$

can be obtained from the following Cholesky decomposition of the covariance matrix $\Sigma$ :

$$
\sigma \cdot \sigma^{\prime}=\Sigma=\operatorname{diag}(\boldsymbol{\nu}) \mathbf{R} \operatorname{diag}(\boldsymbol{\nu})
$$


where $\boldsymbol{\nu}=\left(\nu_{1}, \nu_{2}, \ldots, \nu_{k}\right)$ is a vector of index volatilities, $\operatorname{diag}(\mathbf{v})$ is a diagonal matrix with $\boldsymbol{\nu}$ as diagonal elements, and $\mathbf{R}$ is the correlation matrix. In matrix form, Equation (4) can be expressed as

$$
\left(\begin{array}{c}
R_{j}^{(1)} \\
R_{j}^{(2)} \\
\vdots \\
R_{j}^{(k)}
\end{array}\right)=\left(\begin{array}{c}
f_{j}-\frac{1}{2} \nu_{1}^{2} \\
f_{j}-\frac{1}{2} \nu_{2}^{2} \\
\vdots \\
f_{j}-\frac{1}{2} \nu_{k}^{2}
\end{array}\right) \Delta+\sigma\left(\begin{array}{c}
Z_{j}^{(1)} \\
Z_{j}^{(2)} \\
\vdots \\
Z_{j}^{(k)}
\end{array}\right) \sqrt{\Delta} .
$$

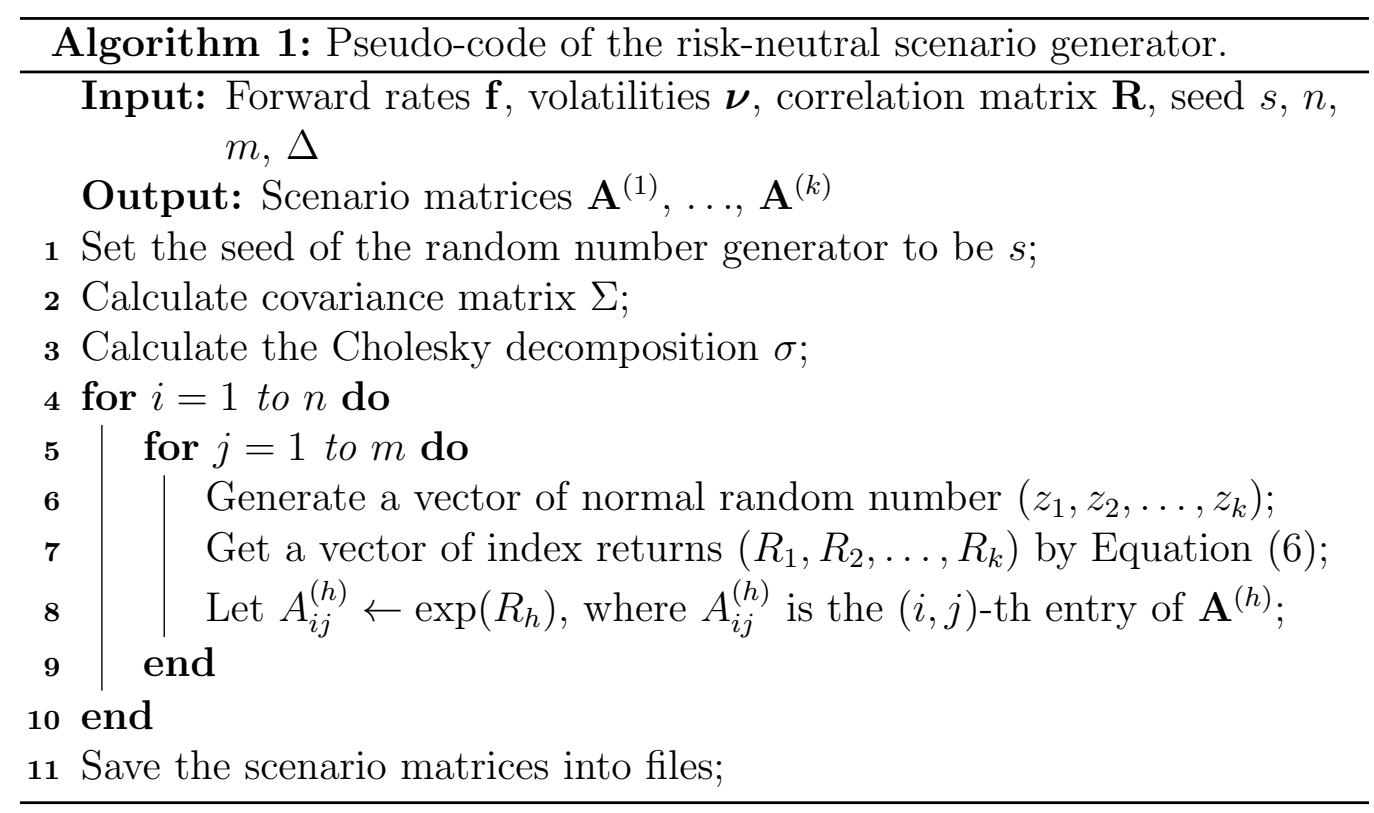

Algorithm 1 shows the pseudo-code of the risk-neutral scenario generator. Once we have index scenarios simulated from Equation (3), we can obtain the investment fund scenarios by blending these index scenarios as follows:

$$
F_{i j}^{(h)}=\sum_{l=1}^{k} w_{h l} A_{i j}^{(l)}, \quad h=1,2, \ldots, g,
$$

where $g$ is the number of investment funds and

$$
W=\left(\begin{array}{cccc}
w_{11} & w_{12} & \cdots & w_{1 k} \\
w_{21} & w_{22} & \cdots & w_{2 k} \\
\vdots & \vdots & \ddots & \vdots \\
w_{g 1} & w_{g 2} & \cdots & w_{g k}
\end{array}\right)
$$


is the fund mapping that maps the $k$ indices to the $g$ investment funds.

\subsection{Real-World Scenario Generator}

Real-world scenarios are used in the outer loop to simulate the movements of the market. Risk-neutral scenarios are prospective and parameters of the risk-neutral scenario generator are calibrated to market data. Realworld scenarios are retrospective and the parameters of a real-world scenario generator are calibrated to historical data.

In practice, the regime-switching model (Hardy, 2001) is typically used to generate real-world scenarios. Here we introduce a multivariate tworegime regime-switching model for generating correlated real-world scenarios for multiple indices. Within a regime and a time period, the evolution of the indices follows the multivariate log-normal model.

Let $\rho_{t}$ denote the regime at time $t$ and $M$ denote the transition matrix, i.e.,

$$
M=\left(\begin{array}{ll}
p_{1,1} & p_{1,2} \\
p_{2,1} & p_{2,2}
\end{array}\right)
$$

where

$$
p_{s, r}=P\left(\rho_{t+1}=r \mid \rho_{t}=s\right), \quad, s, r=1,2 .
$$

Let $\pi=\left(\pi_{1}, \pi_{2}\right)$ be the unconditional probability distribution of the regimeswitching process. Then we have

$$
\pi M=\pi
$$

which gives (Hardy, 2001):

$$
\pi_{1}=\frac{p_{2,1}}{p_{1,2}+p_{2,1}}, \quad \pi_{2}=\frac{p_{1,2}}{p_{1,2}+p_{2,1}} .
$$

Let $S^{(1)}, S^{(2)}, \ldots, S^{(k)}$ be $k$ indices in the financial market. Under the multivariate two-regime regime-switching log-normal model, the risk-world dynamics of the $k$ indices are given by:

$$
\left.S_{t}^{(h)}\right|_{\rho_{t}}=\exp \left[\mu_{h}^{\left(\rho_{t}\right)} t+\sum_{l=1}^{k} \sigma_{h l}^{\left(\rho_{t}\right)} B_{t}^{(l)}\right], \quad S_{0}^{(h)}=1, \quad h=1,2, \ldots, k,
$$

where $B_{t}^{(1)}, B_{t}^{(2)}, \ldots, B_{t}^{(k)}$ are independent standard Brownian motions, $\mu_{h}^{\left(\rho_{t}\right)}$ is the geometric mean of the $h$ th index in the regime $\rho_{t}$, the matrix $\left(\sigma_{h l}^{\left(\rho_{t}\right)}\right)$ 
is used to capture the correlation among the indices in the regime $\rho_{t}$, and $\rho_{t} \in\{1,2\}$ is the regime number.

Let $t_{0}=0, t_{1}=\Delta, \ldots, t_{m}=m \Delta$ be time steps, where $\Delta$ is the time step. Then for $j=1,2, \ldots, m$, the accumulation factor of the $h$ th index for the period $\left(t_{j-1}, t_{j}\right)$ in the regime $\rho_{j}$ can be calculated as

$$
\left.A_{j}^{(h)}\right|_{\rho_{j}}=\frac{\left.S_{j \Delta}^{(h)}\right|_{\rho_{j}}}{\left.S_{(j-1) \Delta}^{(h)}\right|_{\rho_{j}}}=\exp \left[\mu_{h}^{\left(\rho_{t}\right)} \Delta+\sum_{l=1}^{k} \sigma_{h l}^{\left(\rho_{t}\right)} \sqrt{\Delta} Z_{j}^{(l)}\right],
$$

where

$$
Z_{j}^{(l)}=\frac{B_{j \Delta}^{(l)}-B_{(j-1) \Delta}^{(l)}}{\sqrt{\Delta}} .
$$

In matrix form, the returns can be expressed as

$$
\left.\left(\begin{array}{c}
R_{j}^{(1)} \\
R_{j}^{(2)} \\
\vdots \\
R_{j}^{(k)}
\end{array}\right)\right|_{\rho_{j}}=\left.\left(\begin{array}{c}
\ln A_{j}^{(1)} \\
\ln A_{j}^{(2)} \\
\vdots \\
\ln A_{j}^{(k)}
\end{array}\right)\right|_{\rho_{j}}=\left(\begin{array}{c}
\mu_{1}^{\left(\rho_{j}\right)} \\
\mu_{2}^{\left(\rho_{j}\right)} \\
\vdots \\
\mu_{k}^{\left(\rho_{j}\right)}
\end{array}\right) \Delta+\sigma^{\left(\rho_{j}\right)}\left(\begin{array}{c}
Z_{j}^{(1)} \\
Z_{j}^{(2)} \\
\vdots \\
Z_{j}^{(k)}
\end{array}\right) \sqrt{\Delta}
$$

where the matrix $\sigma^{\left(\rho_{j}\right)}$ can be obtained from the following Cholesky decomposition of the covariance matrix $\Sigma^{\left(\rho_{j}\right)}$ :

$$
\sigma^{\left(\rho_{j}\right)} \cdot \sigma^{\left(\rho_{j}\right)^{\prime}}=\Sigma^{\left(\rho_{j}\right)}=\operatorname{diag}\left(\boldsymbol{\nu}^{\left(\rho_{j}\right)}\right) \mathbf{R}^{\left(\rho_{j}\right)} \operatorname{diag}\left(\boldsymbol{\nu}^{\left(\rho_{j}\right)}\right)
$$

where $\boldsymbol{\nu}^{\left(\rho_{j}\right)}$ is a vector of index volatilities for regime $\rho_{j}, \operatorname{diag}\left(\mathbf{v}^{\left(\rho_{j}\right)}\right)$ is a diagonal matrix with $\boldsymbol{\nu}^{\left(\rho_{j}\right)}$ as diagonal elements, and $\mathbf{R}^{\left(\rho_{j}\right)}$ is the correlation matrix for regime $\rho_{j}$.

Let $\rho_{0}$ be the initial regime. Then for $j=1,2, \ldots, m$, the regime for period $j$ can be determined by generating a uniform random number $u$ as follows:

$$
\rho_{j}= \begin{cases}2, & \text { if } \rho_{j-1}=1 \text { and } u \leq p_{1,2} \\ 1, & \text { if } \rho_{j-1}=1 \text { and } u>p_{1,2} \\ 1, & \text { if } \rho_{j-1}=2 \text { and } u \leq p_{2,1} \\ 2, & \text { if } \rho_{j-1}=2 \text { and } u>p_{2,1}\end{cases}
$$




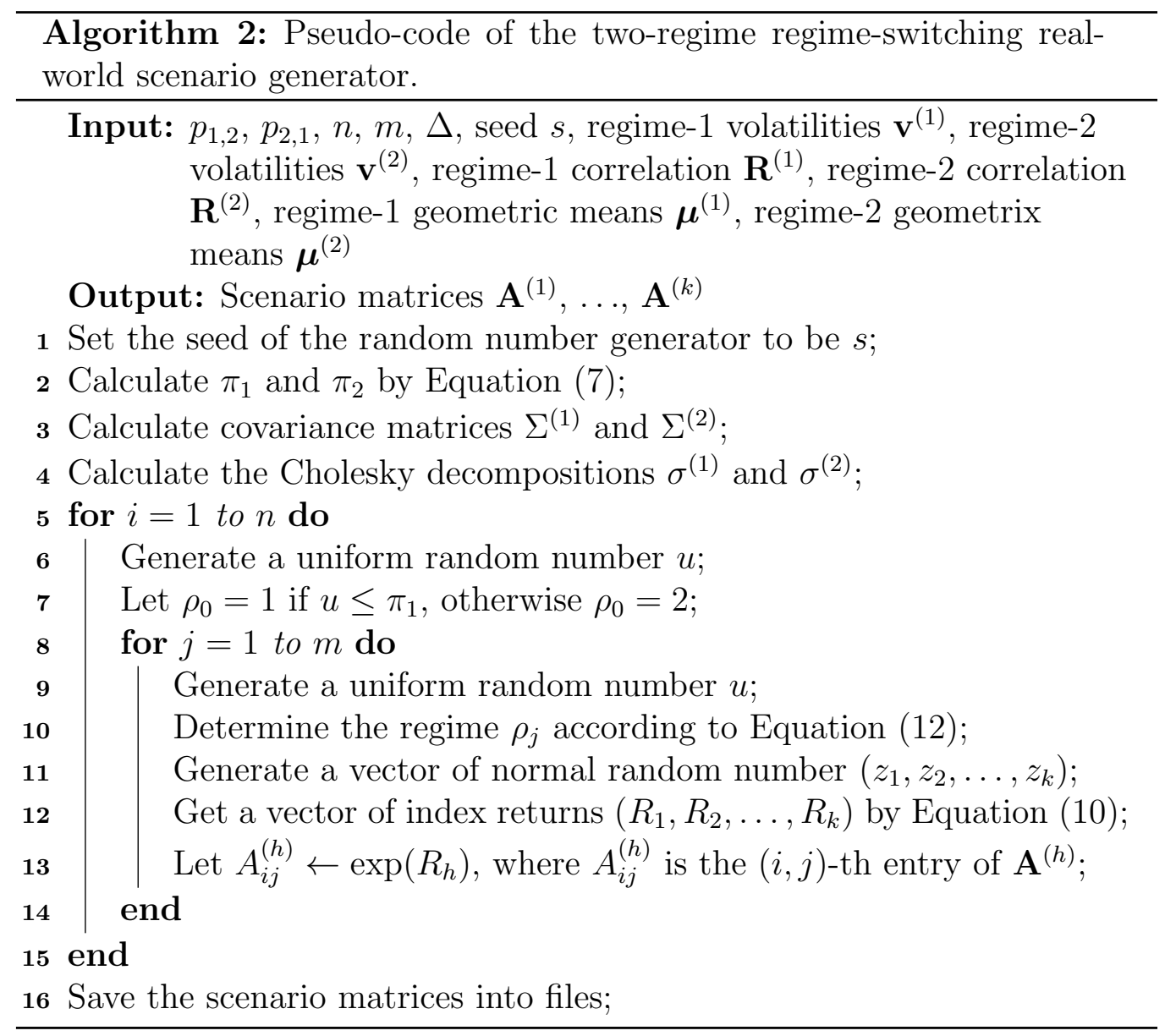

The continuous return of the $h$ th index for the period $\left(t_{j-1}, t_{j}\right)$ in the regime $\rho_{j}$ is calculated

$$
\left.R_{j}^{(h)}\right|_{\rho_{j}}=\left.\ln A_{j}^{(h)}\right|_{\rho_{j}}=\mu_{h}^{\left(\rho_{t}\right)} \Delta+\sum_{l=1}^{k} \sigma_{h l}^{\left(\rho_{t}\right)} \sqrt{\Delta} Z_{j}^{(l)} .
$$

From the above equation, we can derive the expectations and covariances of the conditioned returns as follows:

$$
E\left[\left.R_{j}^{(h)}\right|_{\rho_{j}}\right]=\mu_{h}^{\left(\rho_{t}\right)} \Delta
$$


and

$$
\operatorname{Cov}\left(\left.R_{j}^{(h)}\right|_{\rho_{j}},\left.R_{j}^{(m)}\right|_{\rho_{j}}\right)=\sum_{l=1}^{k} \sigma_{h l}^{\left(\rho_{t}\right)} \sigma_{m l}^{\left(\rho_{t}\right)} \Delta .
$$

The return of the $h$ th index for the period $\left(t_{j-1}, t_{j}\right)$ can be expressed as

$$
R_{j}^{(h)}=\left.R_{j}^{(h)}\right|_{\rho_{j}=1} I_{\left\{\rho_{j}=1\right\}}+\left.R_{j}^{(h)}\right|_{\rho_{j}=2} I_{\left\{\rho_{j}=2\right\}},
$$

where $I$ is an indicator function.

The expected return for the period $\left(t_{j-1}, t_{j}\right)$ can be calculated as (Gan et al., 2014, p177):

$$
\begin{aligned}
E\left[R_{j}^{(h)}\right] & =E\left[\left.R_{j}^{(h)}\right|_{\rho_{j}=1}\right] P\left(\rho_{j}=1\right)+E\left[\left.R_{j}^{(h)}\right|_{\rho_{j}=2}\right] P\left(\rho_{j}=2\right) \\
& =\mu_{h}^{(1)} \Delta \pi_{1}+\mu_{h}^{(2)} \Delta \pi_{2} .
\end{aligned}
$$

From Equation (14), we have

$$
R_{j}^{(h)} R_{j}^{(m)}=\left.\left.R_{j}^{(h)}\right|_{\rho_{j}=1} R_{j}^{(m)}\right|_{\rho_{j}=1} I_{\left\{\rho_{j}=1\right\}}+\left.\left.R_{j}^{(h)}\right|_{\rho_{j}=2} R_{j}^{(m)}\right|_{\rho_{j}=2} I_{\left\{\rho_{j}=2\right\}},
$$

which gives

$$
\begin{aligned}
E\left[R_{j}^{(h)} R_{j}^{(m)}\right]= & \pi_{1}\left(\mu_{h}^{(1)} \mu_{m}^{(1)} \Delta^{2}+\sum_{l=1}^{k} \sigma_{h l}^{(1)} \sigma_{m l}^{(1)} \Delta\right)+ \\
& \pi_{2}\left(\mu_{h}^{(2)} \mu_{m}^{(2)} \Delta^{2}+\sum_{l=1}^{k} \sigma_{h l}^{(2)} \sigma_{m l}^{(2)} \Delta\right)
\end{aligned}
$$

Therefore, we have

$$
\begin{aligned}
\operatorname{Cov}\left(R_{j}^{(h)}, R_{j}^{(m)}\right)= & E\left[R_{j}^{(h)} R_{j}^{(m)}\right]-E\left[R_{j}^{(h)}\right] E\left[R_{j}^{(m)}\right] \\
= & \pi_{1} \pi_{2} \Delta^{2}\left(\mu_{h}^{(1)}-\mu_{h}^{(2)}\right)\left(\mu_{m}^{(1)}-\mu_{m}^{(2)}\right)+ \\
& \pi_{1} \sum_{l=1}^{k} \sigma_{h l}^{(1)} \sigma_{m l}^{(1)} \Delta+\pi_{2} \sum_{l=1}^{k} \sigma_{h l}^{(2)} \sigma_{m l}^{(2)} \Delta .
\end{aligned}
$$


Letting $m=h$ in the above equation, we get the variance of the return as follows:

$$
\begin{aligned}
& \operatorname{Var}\left(R_{j}^{(h)}\right) \\
= & E\left[\left(R_{j}^{(h)}\right)^{2}\right]-\left(E\left[R_{j}^{(h)}\right]\right)^{2} \\
= & \pi_{1} \pi_{2} \Delta^{2}\left(\mu_{h}^{(1)}-\mu_{h}^{(2)}\right)^{2}+\pi_{1} \sum_{l=1}^{k}\left(\sigma_{h l}^{(1)}\right)^{2} \Delta+\pi_{2} \sum_{l=1}^{k}\left(\sigma_{h l}^{(2)}\right)^{2} \Delta \\
= & \pi_{1} \pi_{2} \Delta^{2}\left(\mu_{h}^{(1)}-\mu_{h}^{(2)}\right)^{2}+\pi_{1} \Delta\left(\nu_{h}^{(1)}\right)^{2}+\pi_{2} \Delta\left(\nu_{h}^{(2)}\right)^{2},
\end{aligned}
$$

where $\nu_{h}^{\left(\rho_{j}\right)}$ is the volatility of the $h$ th index in the regime $\rho_{j}$, i.e.,

$$
\nu_{h}^{\left(\rho_{j}\right)}=\sqrt{\sum_{l=1}^{k}\left(\sigma_{h l}^{\left(\rho_{j}\right)}\right)^{2}} .
$$

Equations (15) and (17) can be used to validate the real-world scenarios. These equations can also be used to specify parameters for the two-regime regime-switching model if we want to control the overall mean returns and the overall volatilities of the indices.

\subsection{Nested Stochastic Valuation}

To describe how nested stochastic projections are done, we let $N_{1}$ be the number of outer loop paths and let $T_{1}$ be the number of time nodes in the outer loop. Let $X=\left\{\mathbf{x}_{1}, \mathbf{x}_{2}, \ldots, \mathbf{x}_{n}\right\}$ be a portfolio of $n$ VA policies. Algorithm 3 shows a high-level sketch of the nested stochastic valuation engine. At each node along each outer loop path, we calculate the fair market values of each policy using the risk-neutral scenarios. For details about how policies are aged along a real-world path and how the cash flows are projected along a risk-neutral path, readers are referred to Gan and Valdez (2017).

To assess the performance of dynamic hedging, partial dollar deltas are required as hedging is done by individual tradable indices. The partial dollar delta on the $h$ th index is normally calculated as follows:

$\frac{F M V\left(\ldots, A V_{h-1}, 1.01 A V_{h}, A V_{h+1}, \ldots\right)-F M V\left(\ldots, A V_{h-1}, 0.99 A V_{h}, A V_{h+1}, \ldots\right)}{0.02}$ 


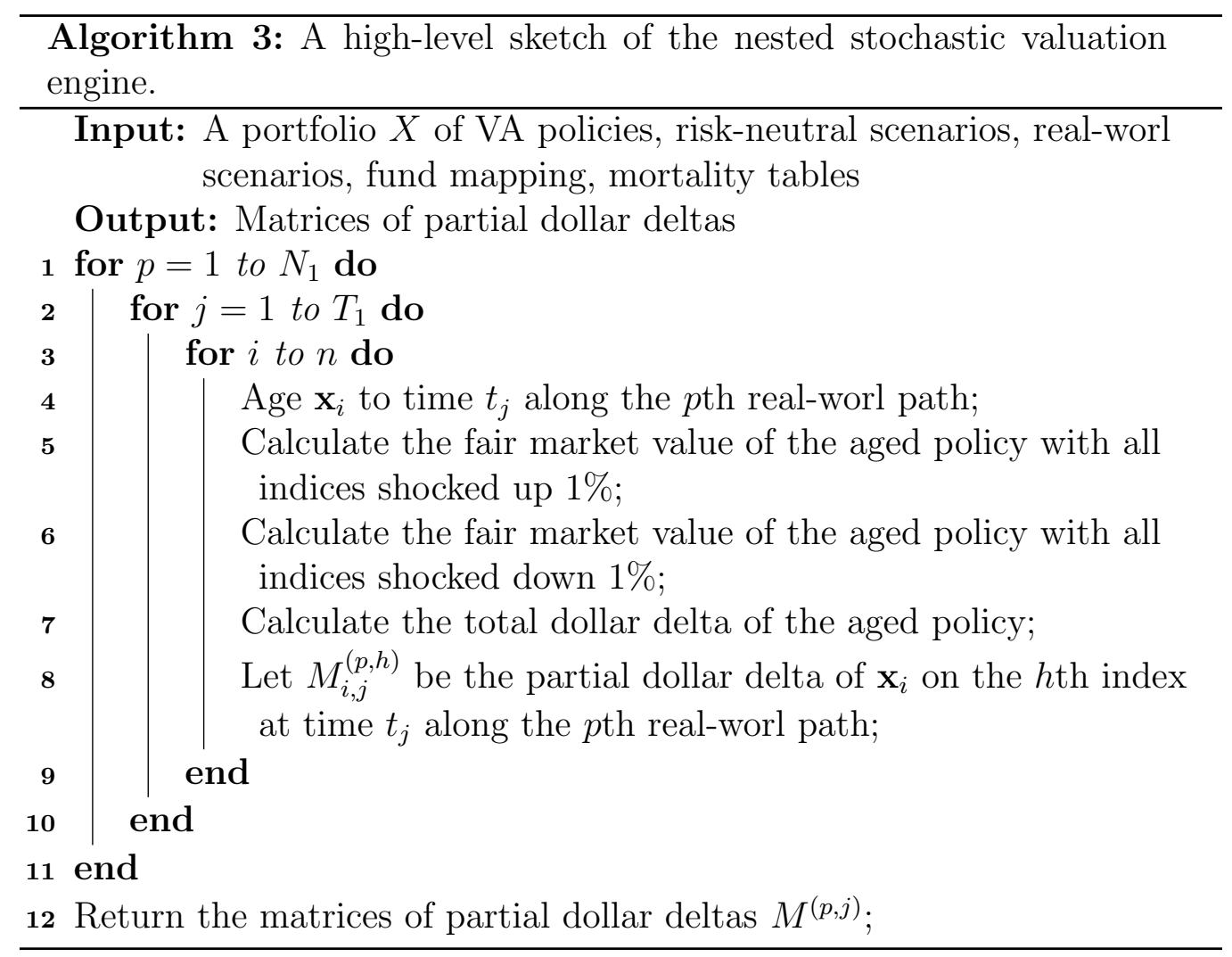

where $A V_{h}$ denotes the account value invested in the $h$ th index. However, calculating partial dollar deltas using the above equation requires projecting cash flows at many index shocks. This is prohibitive under the nested stochastic valuation framework.

To reduce the runtime, we only calculate total dollar delta at each node along an outer loop path as follows:

$$
\Delta=\frac{F M V\left(1.01 A V_{1}, \ldots, 1.01 A V_{k}\right)-F M V\left(0.99 A V_{1}, \ldots, 0.99 A V_{k}\right)}{0.02}
$$

where $k$ is the number of indices. Then we approximate the partial dollar deltas as follows:

$$
\Delta^{(h)}=\frac{A V_{h}}{A V_{1}+\cdots+A V_{k}} \Delta .
$$

The relation given in Equation (18) can be derived as follows. Suppose that the fair market value of the guarantees embedded in a VA policy is a function 
of the total account value, i.e.,

$$
F M V=f\left(A V_{1}+A V_{2}+\cdots+A V_{k}\right) .
$$

Then the partial dollar delta on the $h$ th index is calculated as

$$
\Delta^{(h)}=\frac{\partial f}{\partial A V_{h}} A V_{h}=\frac{\partial f}{\partial T A} \cdot \frac{\partial T A}{\partial A V_{h}} \cdot A V_{h}=\frac{\partial f}{\partial T A} \cdot T A \cdot \frac{A V_{h}}{T A}=\Delta \cdot \frac{A V_{h}}{T A},
$$

where $T A=A V_{1}+\cdots A V_{k}$ is the total account value.

\section{Synthetic Portfolio and Payoffs}

In this section, we describe the synthetic portfolio and the payoffs of the guarantees embedded in the VA policies.

\subsection{Synthetic Portfolio}

We adopted a subset of the synthetic VA portfolio created in Gan and Valdez (2017). That synthetic portfolio contains 19 types of products, each of which has 10,000 policies. We selected 2,000 policies from each product type. The subset contains 38,000 policies. Readers are referred to Gan and Valdez (2017) for a description of the features or variables of the VA policies.

Table 1: Distribution of gender by product type.

\begin{tabular}{llllllll}
\hline Gender & ABRP & ABRU & ABSU & DBAB & DBIB & DBMB & DBRP \\
\hline F & 779 & 768 & 809 & 787 & 759 & 833 & 805 \\
M & 1221 & 1232 & 1191 & 1213 & 1241 & 1167 & 1195 \\
\hline Gender & DBRU & DBSU & DBWB & IBRP & IBRU & IBSU & MBRP \\
\hline F & 785 & 782 & 789 & 782 & 826 & 824 & 775 \\
M & 1215 & 1218 & 1211 & 1218 & 1174 & 1176 & 1225 \\
\hline Gender & MBRU & MBSU & WBRP & WBRU & WBSU & & \\
\hline F & 798 & 798 & 813 & 812 & 781 & & \\
M & 1202 & 1202 & 1187 & 1188 & 1219 & & \\
\hline
\end{tabular}

Table 1 shows the number of policies in each product type by gender. About $40 \%$ of the policies in each product are female. Table 2 shows the 
Table 2: Summary statistics of some fields. Note that age and ttm are calculated from the birth date, valuation date, and maturity date.

\begin{tabular}{lrrrrr}
\hline & Min & 1st Q & Mean & 3rd Q & Max \\
\hline gbAmt & 0.00 & $187,601.23$ & $327,213.71$ & $446,403.84$ & $1,060,311.72$ \\
gmwbBalance & 0.00 & 0.00 & $35,501.02$ & 0.00 & $499,708.73$ \\
withdrawal & 0.00 & 0.00 & $22,605.36$ & 0.00 & $499,585.73$ \\
FundValue1 & 0.00 & 0.00 & $33,632.30$ & $50,083.85$ & $798,936.37$ \\
FundValue2 & 0.00 & 0.00 & $38,673.18$ & $57,221.55$ & $1,026,213.34$ \\
FundValue3 & 0.00 & 0.00 & $26,778.14$ & $39,154.69$ & $752,945.34$ \\
FundValue4 & 0.00 & 0.00 & $26,231.25$ & $39,331.61$ & $566,338.64$ \\
FundValue5 & 0.00 & 0.00 & $22,768.91$ & $34,841.42$ & $481,399.12$ \\
FundValue6 & 0.00 & 0.00 & $35,386.64$ & $52,585.38$ & $1,042,335.65$ \\
FundValue7 & 0.00 & 0.00 & $29,898.18$ & $44,511.19$ & $806,540.12$ \\
FundValue8 & 0.00 & 0.00 & $30,303.87$ & $45,505.06$ & $704,720.85$ \\
FundValue9 & 0.00 & 0.00 & $29,983.68$ & $44,034.63$ & $851,307.63$ \\
FundValue10 & 0.00 & 0.00 & $30,092.13$ & $45,276.64$ & $691,822.70$ \\
age & 34.52 & 42.11 & 49.56 & 56.96 & 64.46 \\
ttm & 0.59 & 10.26 & 14.49 & 18.68 & 28.52 \\
\hline
\end{tabular}


summary statistics of some numerical fields. From the table, we see that all funds have many zeros. This is because many policies generally do not invest in all the funds. The age is the number of years between the birth date and the current date. The time to maturity is calculated from the current date and the maturity date.

\subsection{Guarantee Payoffs}

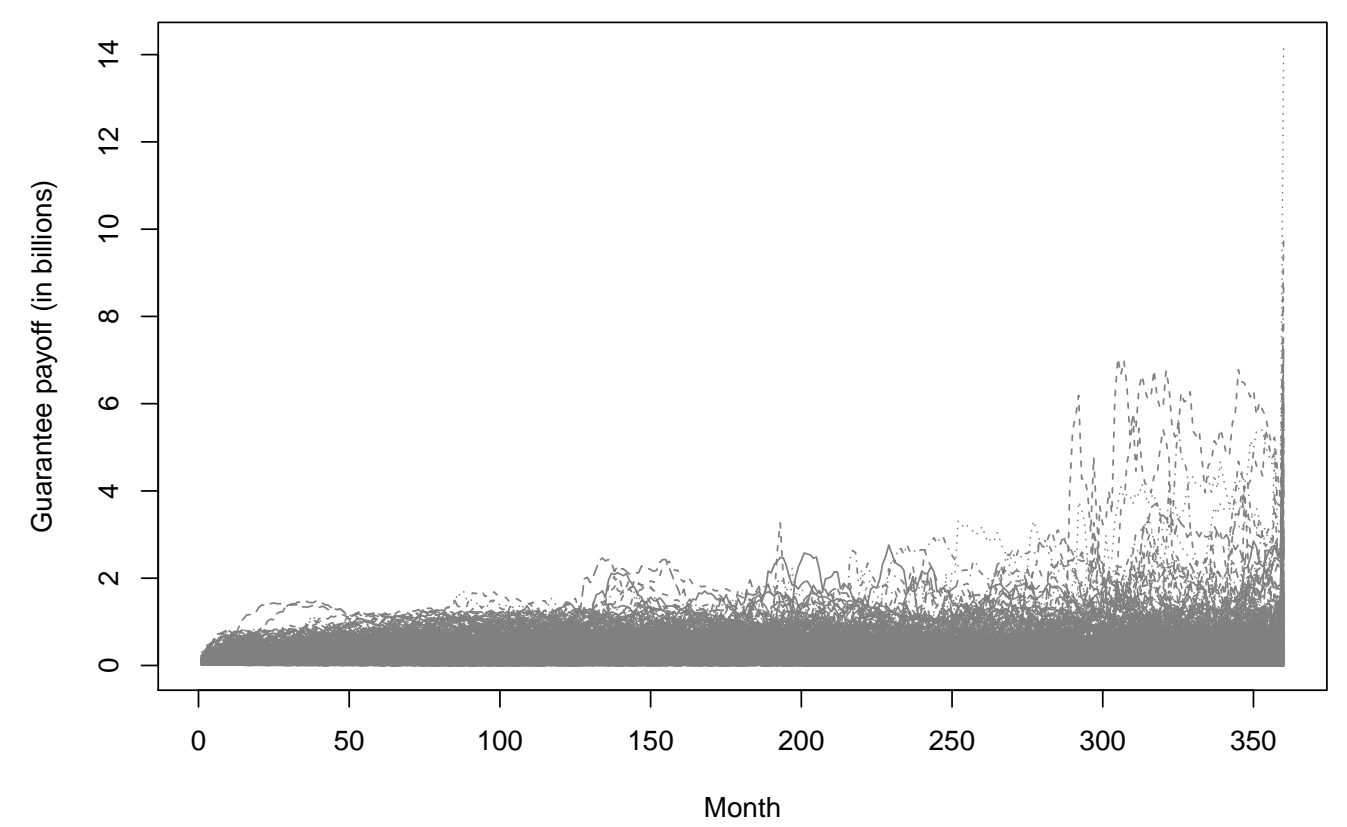

Figure 3: Guarantee payoffs along the 1,000 real-world paths.

We calculated the payoffs of the guarantees for the portfolio along each of the 1,000 real-world path. The payoff is calculated as the sum of the death benefit and the living benefit. Figure 3 shows the guarantee payoffs of the portfolio at each month along the 1,000 real-world path. From the figure, we see that there are some relatively large guarantee payoffs after the 300th month at some real-world paths. The large payoffs are caused the the GMAB products, which allow policyholders to renew.

We also calculated the present values of the guarantee payoffs along each of the 1,000 real-world paths. Figure 6 shows a histogram of these present values. The histogram shows that the distribution of the present values is positively skewed. At some bad real-world paths, the guarantee payoffs are 


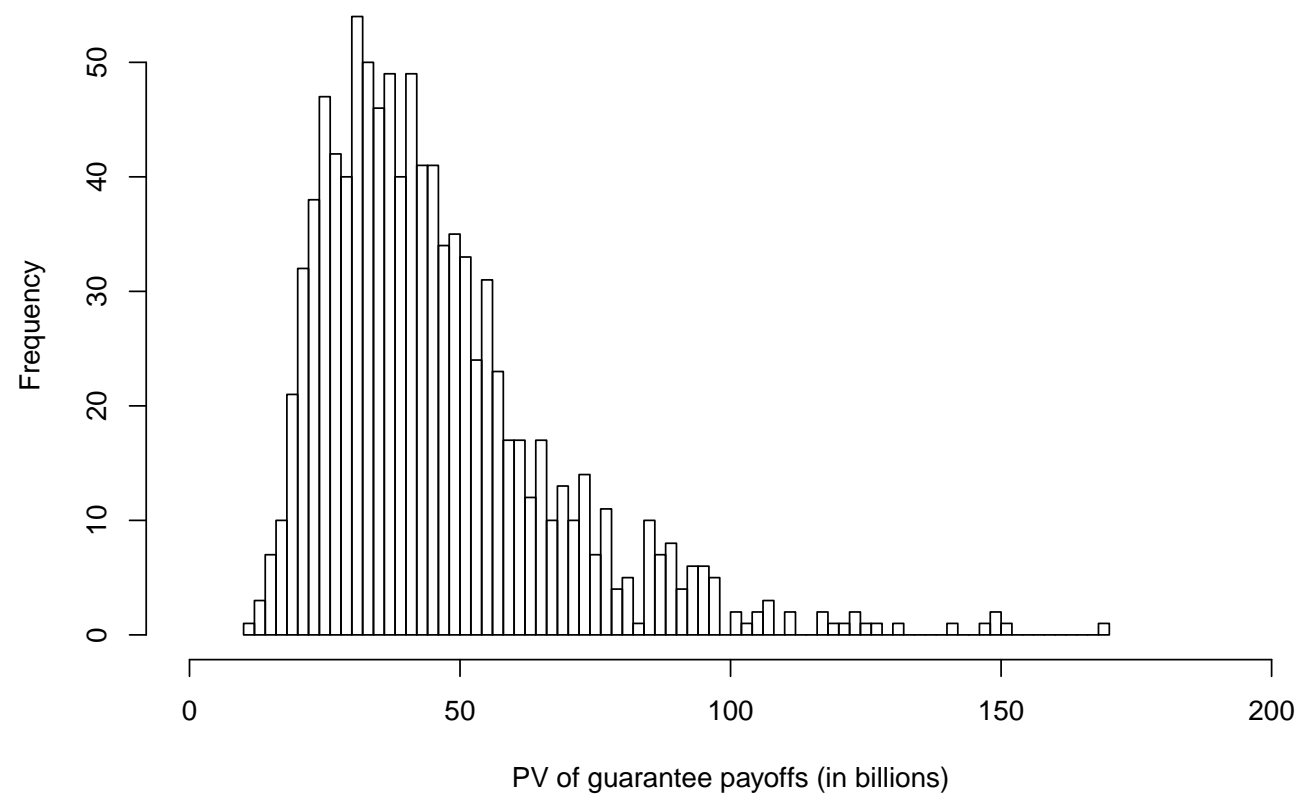

Figure 4: Present values of the guarantee payoffs.

Table 3: Summary statistics and conditional tail expectations of the present values of the guarantee payoffs. The numbers are in millions.

(a)

\begin{tabular}{llllll}
\hline Min. & 1st Qu. & Median & Mean & 3rd Qu. & Max. \\
\hline 11,421 & 30,393 & 40,556 & 45,535 & 54,793 & 168,574 \\
\hline \multicolumn{5}{c}{ (b) } \\
\cline { 2 - 5 } \\
\cline { 2 - 5 } & CTE50 & CTE75 & CTE95 & \\
\hline & $61,332.68$ & $75,594.85$ & $108,113.29$ \\
\hline
\end{tabular}


much larger than those at other real-world paths. Table 3(a) shows some summary statistics of these present values. At the best real-world path, the guarantee payoff of this portfolio is 11,421 millions. If the worst real-world path occurs, the guarantee payoff of this portfolio is 168,574 millions.

Table 3(b) shows the conditional tail expectations (CTEs) of the present values of the guarantee payoffs at three different levels. The CTE75 is calculated as the mean of present values from the worst $0.25 \times 1000=250$ real-world paths. From the table, we see that the CTE75 is around 75,595 millions.
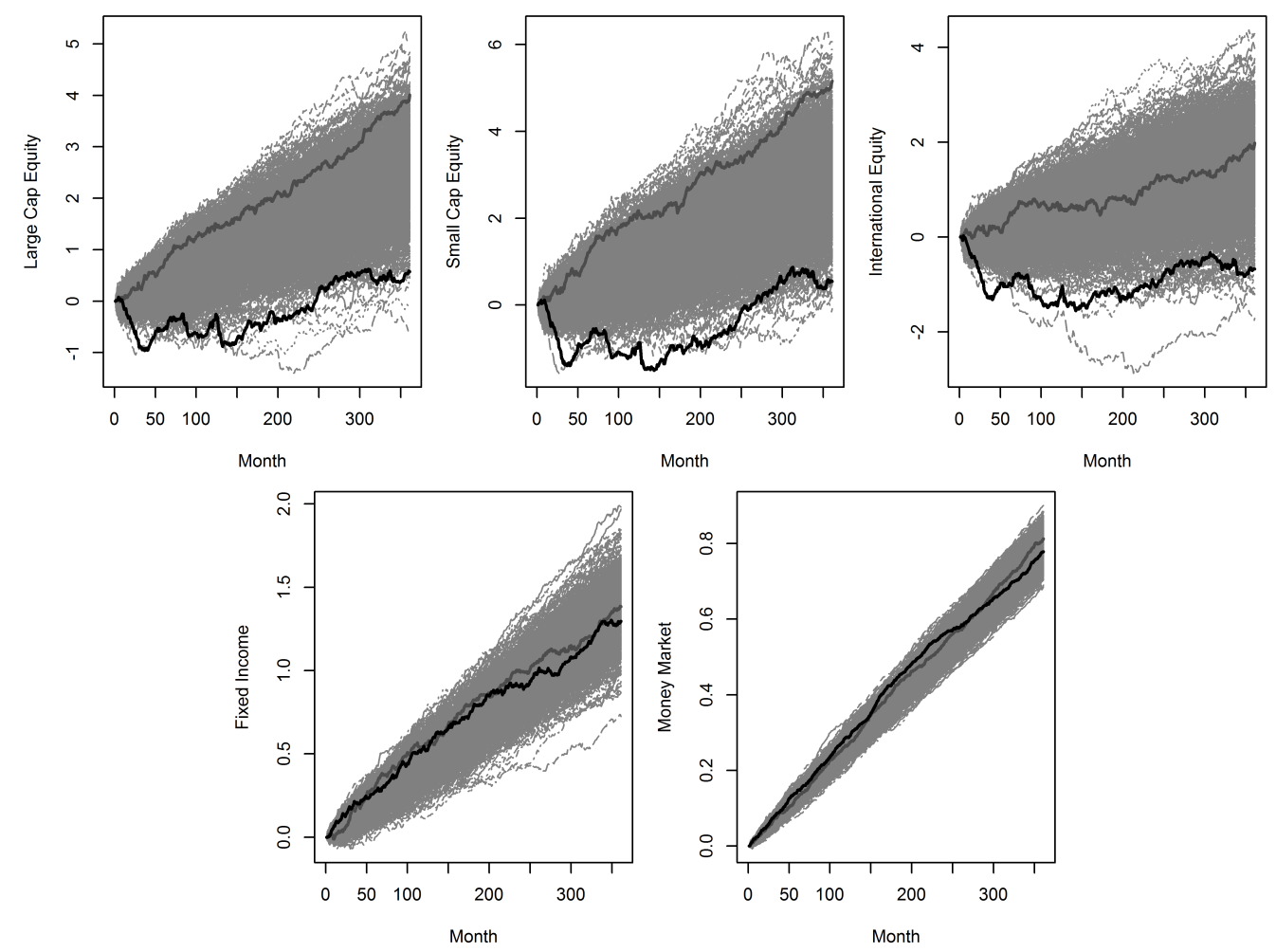

Figure 5: Real-world paths of the indices. The dark thick line is the worst real-world path. The gray thick line is the best real-world path.

Figure 5 shows the 1,000 real-world paths of the five indices, which are the large cap equity, the small cap equity, the international equity, the fixed income, and the money market. The dark thick line in each subfigure corresponds to the worst real-world path, which produces the largest present value of the guarantee payoffs. The gray thick line in each subfigure corresponds 


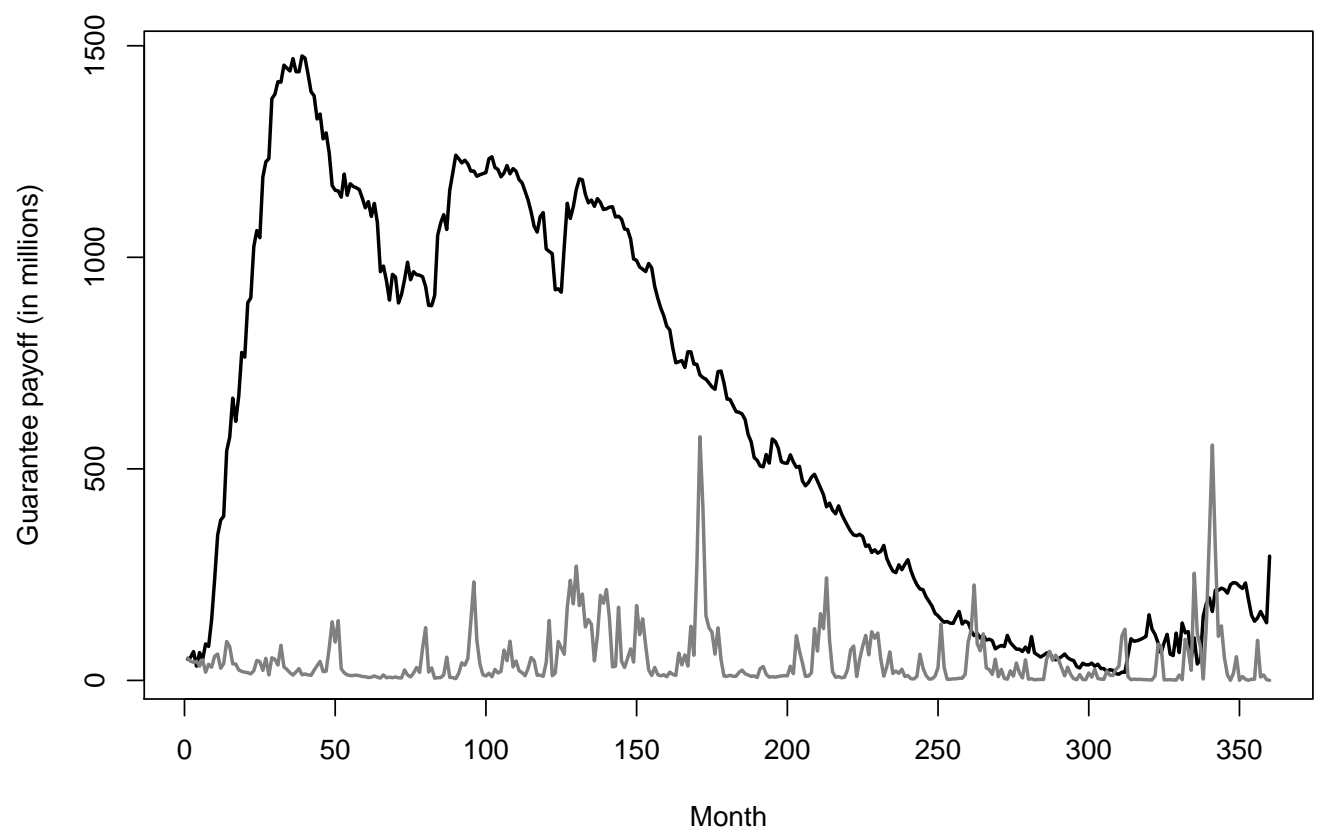

Figure 6: Guarantee payoffs along the worst (the dark line) and the best (the gray line) real-world paths.

to the best real-world path, which produces the lowest present value of the guarantee payoffs. From the figure, we see that the best path is above the worst path.

Note that the best real-world path is not the one at the very top and the worst real-world path is not the one at the very bottom. This is because the payoffs of GMAB products in bull market are large. In other words, if the real-world path at the very top occurs, the GMAB products will incur large payoffs because the policyholders can renew by reseting the benefit to the higher of the account value and the existing benefit base.

Figure 6 shows the guarantee payoffs at monthly steps along the best and the worst real-world paths. From the figure, we see that in general the payoffs along the best real-world path are higher than those along the worst real-world path. At a few months near the end of the projection horizon, the payoffs at the best path are higher than those at the worst path. This is caused by the GMAB products, which have higher payoffs at better markets due to the renew feature. 


\section{Partial Dollar Deltas}

In this section, we present the partial dollar deltas calculated by the nested Monte Carlo simulation method described in Section 2.

As discussed in Section 2, the nested stochastic valuation program produces many matrices of the partial dollar deltas. In fact, the program produces $N_{1} \times H$ matrices of partial dollar deltas, where $N_{1}$ is the number of real-world paths and $H$ is the number of indices. For $p=1,2, \ldots, N_{1}$ and $h=1,2, \ldots, H$, let $M^{(p, h)}$ be the matrix of the partial dollar deltas on the $h$ th index:

$$
M^{(p, h)}=\left(\begin{array}{cccc}
M_{1,1}^{(p, h)} & M_{1,2}^{(p, h)} & \ldots & M_{1, T}^{(p, h)} \\
M_{2,1}^{(p, h)} & M_{2,2}^{(p, h)} & \ldots & M_{2, T}^{(p, h)} \\
\vdots & \vdots & \ddots & \vdots \\
M_{n, 1}^{(p, h)} & M_{n, 2}^{(p, h)} & \ldots & M_{n, T}^{(p, h)}
\end{array}\right)
$$

where $n$ is the number of policies in the portfolio, $T$ is the number of time points where partial dollar deltas are calculated, and $M_{i, j}^{(p, h)}$ denotes the partial dollar delta of the $i$ th policy on the $h$ th index at $j$ th evaluation time point along the $p$ th real-world path. Since we used $N_{1}=1,000$ real-world paths and $T=31$ evaluation time points and the number of indices is $H=5$, the number of matrices we produced is 5,000. Each matrix has a size of $38,000 \times 31$. We saved all the matrices to CSV files with only six decimal places. If zip all the CSV files, the size of the zip file is around 20GB.

\subsection{Aggregate Results}

The aggregate partial dollar deltas along a real-world path are calculated as follows:

$$
M_{j}^{(p, h)}=\sum_{i=1}^{n} M_{i, j}^{(p, h)} .
$$

In other words, the aggregate partial dollar deltas are the partial dollar deltas of the whole portfolio. The aggregate total dollar deltas are calculated as

$$
M_{j}^{(p)}=\sum_{h=1}^{H} \sum_{i=1}^{n} M_{i, j}^{(p, h)} .
$$

Figure 7 shows the aggregate partial dollar deltas and aggregate total dollar deltas along the 1,000 real-world paths. From the figure, we have the following observations: 

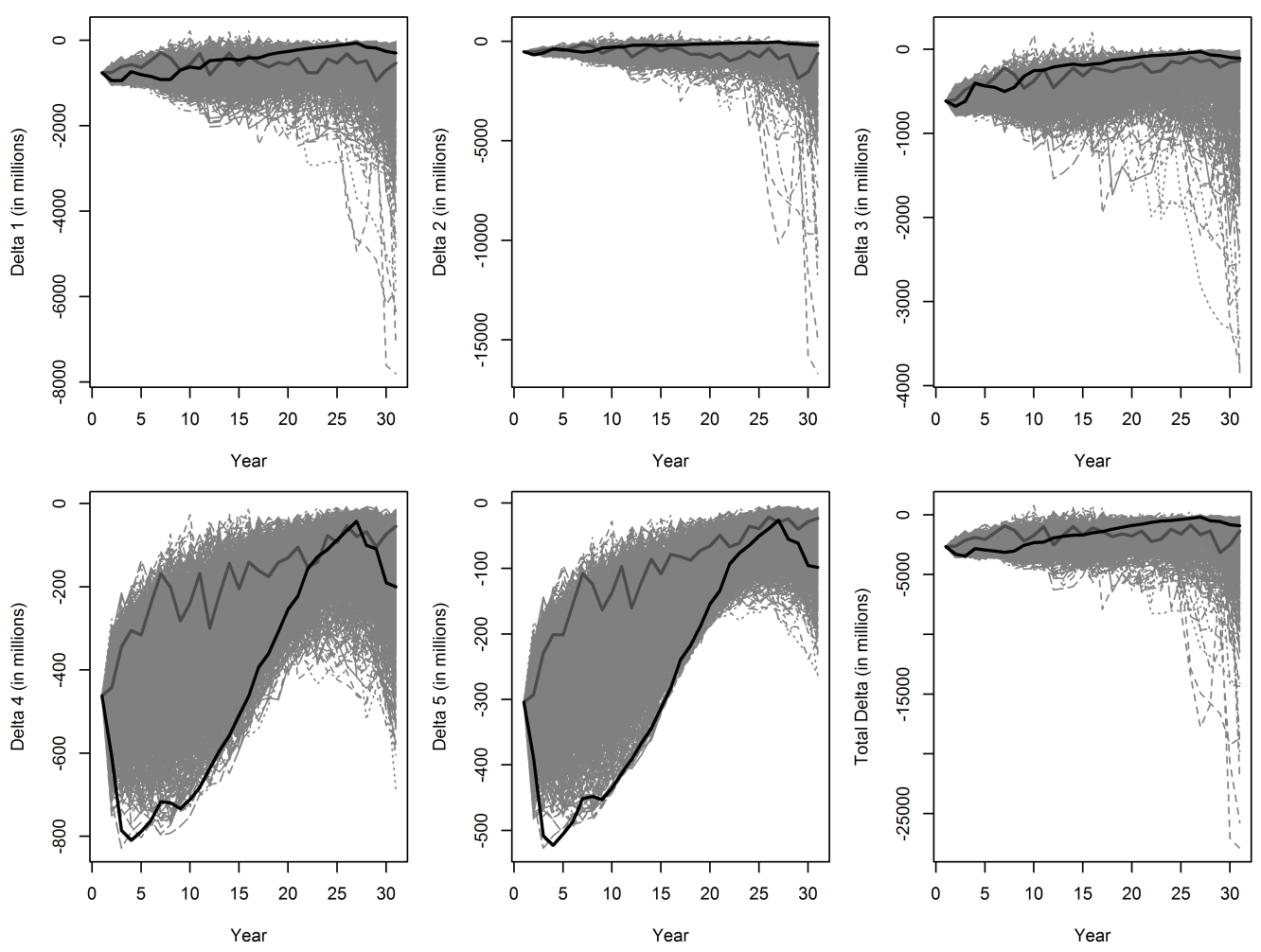

Figure 7: Aggregate partial dollar deltas and aggregate total dollar delta along the 1,000 real-world paths. The dark thick and the gray thick lines correspond to the worst and the best real-world paths, respectively.

- The aggregate partial dollar deltas do not approach zero at the end of the projection horizon. This is caused by the GMAB products, which behavior like call options.

- The guarantees are more sensitive to indices with higher volatilities. For example, the magnitudes of the aggregate partial dollar deltas on the small cap equity are larger than those on other indices.

- The aggregate total dollar deltas along the best and the worst realworld paths have similar magnitudes. This is because the dollar deltas of the GMAB product offset those of other products.

- For equity indices, which have high volatilities, the aggregate partial dollar deltas have similar magnitudes along the best and the worst 
real-world paths. For non-equity indices, which have low volatilities, the aggregate partial dollar deltas along the worst real-world path have higher magnitudes than those along the best real-world path.
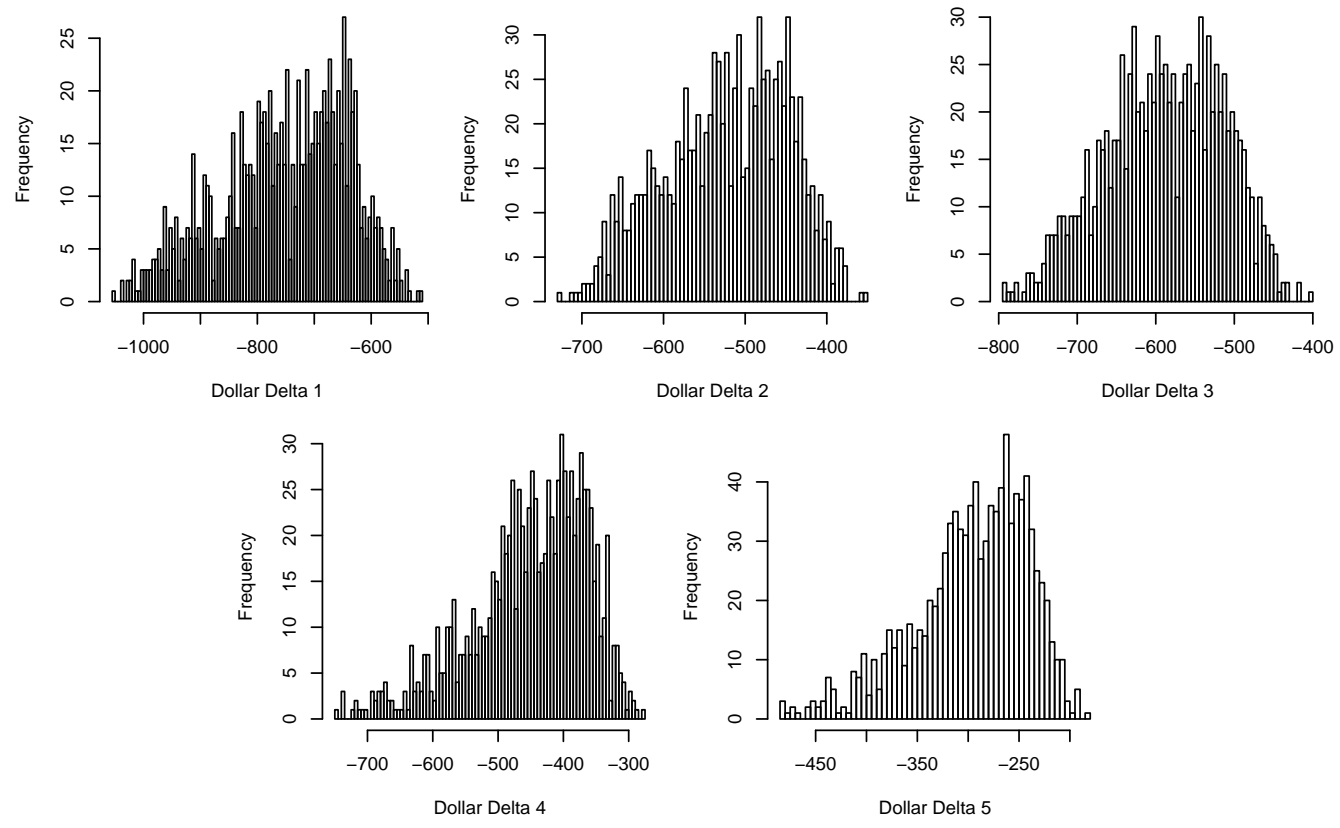

Figure 8: A histogram of the aggregate partial dollar deltas along the 1,000 real-world paths at the end of year 1 . The numbers are in millions.

Figure 8 and Figure 9 shows the histograms of the aggregate partial dollar deltas at the year 1 and the year 30, respectively. From the figures, we see that the distributions of the aggregate partial dollar deltas at the year 30 is more skewed that those at the year 1 .

\subsection{Seriatim Results}

There are many seriatim results, making it difficult to show all the results in details. In this subsection, we only show the seriatim results from the best and the worst real-world paths identified before. Figure 10 shows a histogram of the seriatim partial dollar deltas at the end of year 1 if the best real-world path occurs. Figure 11 shows a similar histogram if the worst real-world path occurs. Both figures show that the distributions of the seriatim partial dollar deltas are highly skewed. In addition, some policies have positive 

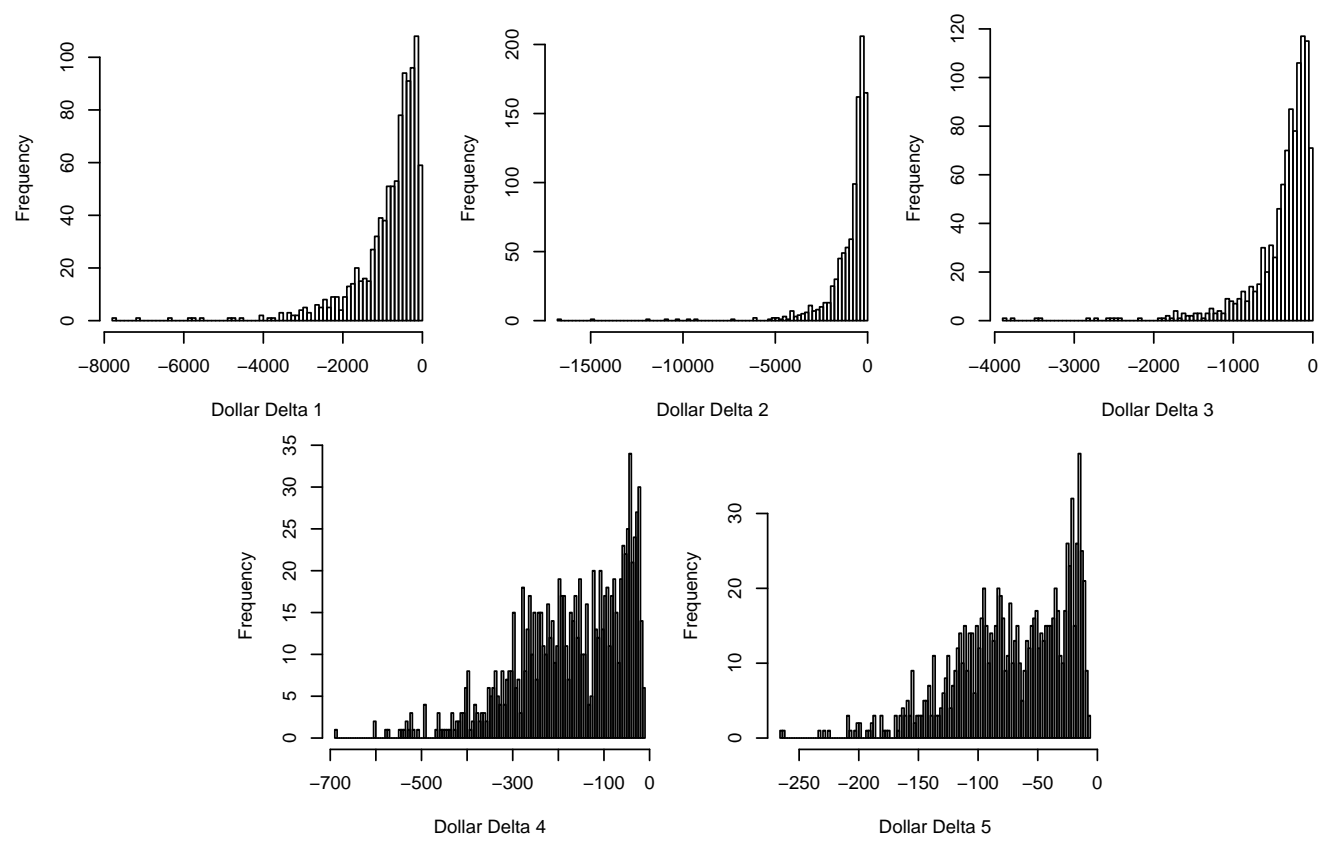

Figure 9: A histogram of the aggregate partial dollar deltas along the 1,000 real-world paths at the end of year 30 . The numbers are in millions.

dollar deltas if the best real-world path occurs. This is caused by the GMAB products as a bull market can trigger the renew option embedded in such products.

Figure 12 and Figure 13 show the box plots of seriatim partial dollar deltas by product type at the end of year 1 along the best and the worst real-world paths, respectively. From these figures, we see that the GMAB, GMIB, and GMMB products are more sensitive than the GMDB and GMWB products in terms of the magnitudes of the deltas. In addition, more policies have positive deltas when the best real-world path occurs than the case when the worst real-world path occurs.

\subsection{Runtime}

We implemented the nested stochastic valuation engine as a distributed multi-threading program in Java. We used the HPC (High Performance Computing) cluster ${ }^{1}$ at the University of Connecticut to run the program.

\footnotetext{
${ }^{1}$ https://hpc.uconn.edu/
} 

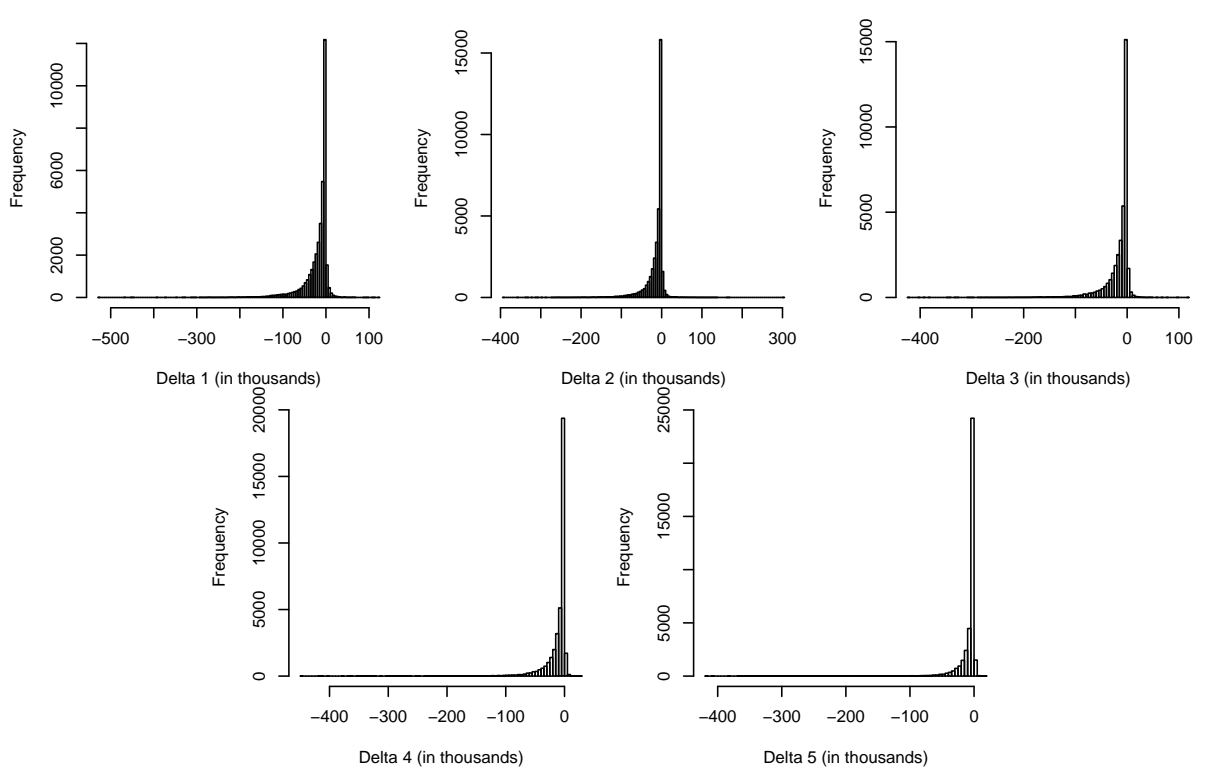

Figure 10: Histograms of the seriatim partial dollar deltas at the end of year 1 of the best real-world path.

In particular, we used 8 instances of the program with 20 cores for each instance to calculate the partial dollar deltas for the portfolio. Each instance of the program handles one outer loop path at a time. The coordination between different instances is done via the mechanism of file locking. Even with 160 cores, it took about two weeks to get all the calculations done.

For the convenience of comparison, we accumulate the runtime used by all threads to get the runtime that would be used by a single core. Figure 14 shows a histogram of the runtime used to calculate the partial dollar deltas for an outer loop path. From the figure, we see that if a single core is used, it would take the core about 20-32 hours to finish the calculation for a single outer loop path. If we aggregate the runtime used to process all the 1,000 outer loop paths, the runtime is $93,722,002.966$ seconds or 2.97 years. In other words, if we use a single CPU to calculate the partial dollar deltas for the portfolio of 38,000 VA policies with 1,000 real-world path and 1,000 risk-neutral paths, it would take this CPU about 2.97 years to finish the calculation. Note that we only calculated the partial dollar deltas at 30 time points along the outer loop paths. If we want to calculate the deltas at 360 time points along the outer loop paths, it will take a single core about 36 years. 

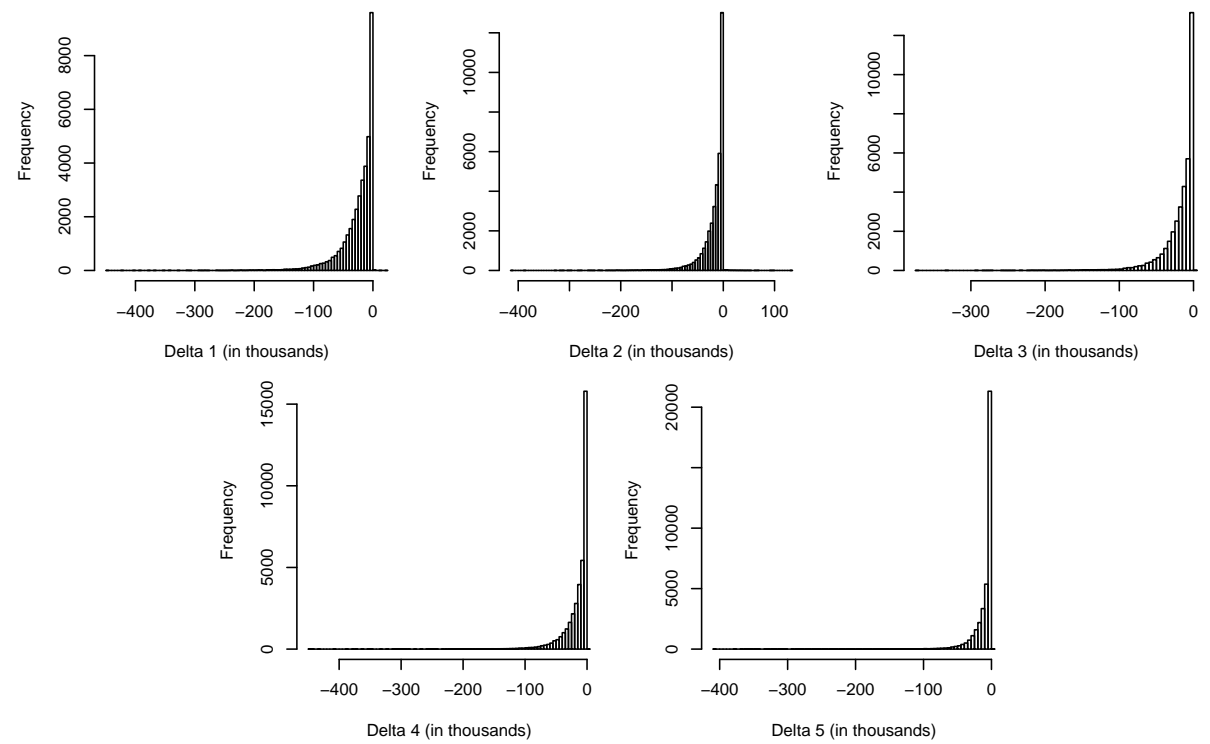

Figure 11: Histograms of the seriatim partial dollar deltas at the end of year 1 of the worst real-world path.

\section{Concluding Remarks}

Metamodeling techniques have been proposed to address the computational issues associated with the nested stochastic valuation of large VA portfolios. However, it is difficult for researchers to obtain real datasets from insurance companies to test the metamodeling techniques and publish the results in academic journals. It is the primary purpose of this paper to create synthetic datasets to address computational issues. These synthetic datasets can be used by researchers and practitioners to test techniques, especially metamodeling techniques, to speed up the nested stochastic valuation of large VA portfolios.

These synthetic datasets have some limitations. First, the synthetic VA policies are simpler than VA policies sold in the real-world. Second, the Monte Carlo simulation is also simpler than the one used in practice. For example, we did not consider the policyholder behavior in the cash flow projections. Although the synthetic datasets have limitations, we can still use them to test metamodeling techniques. If a metamodeling technique does not work for the synthetic datasets, then it is unlikely to work for real datasets. 

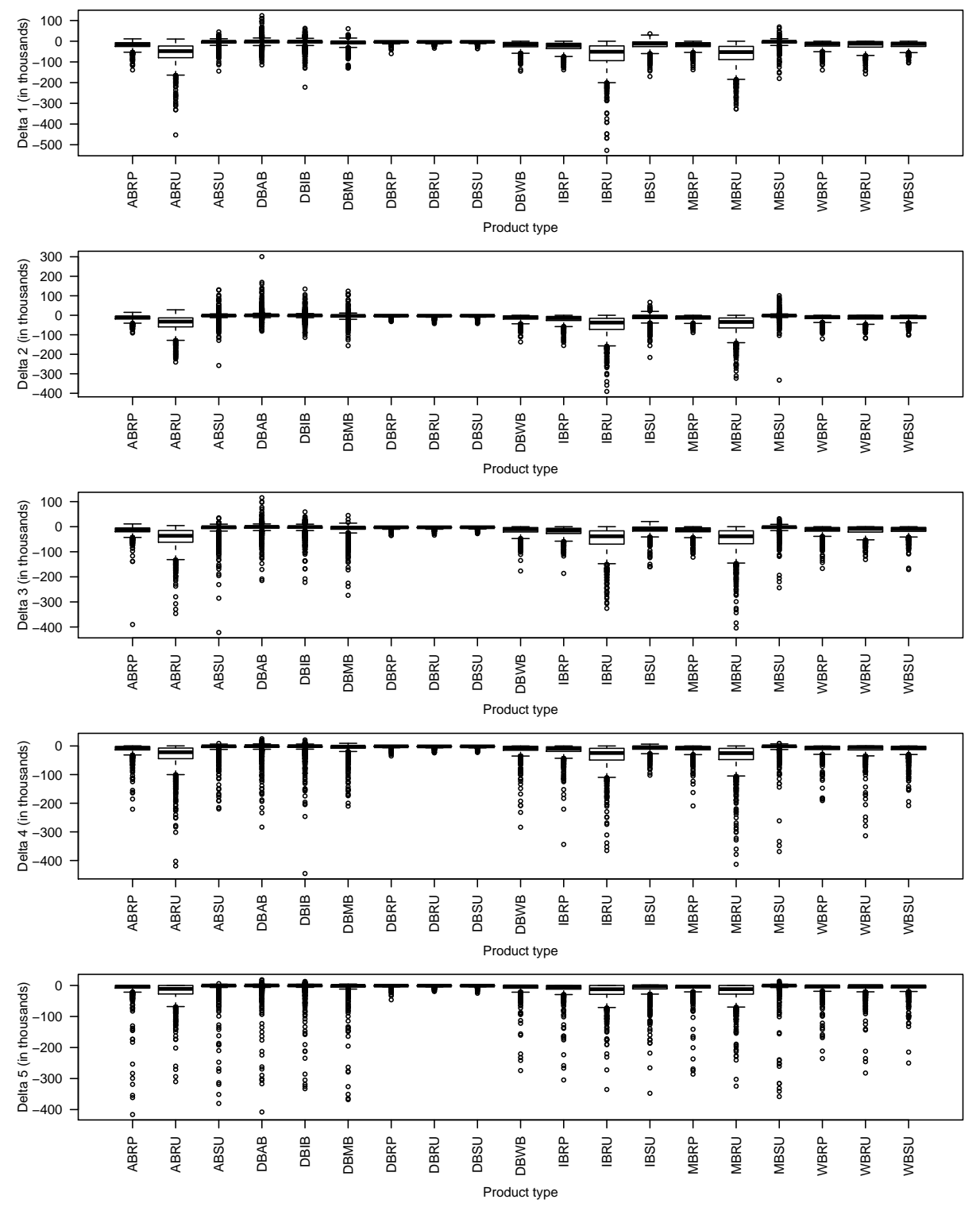

Figure 12: Box plots of the partial dollar deltas by product type at the end of year 1 of the best real-world path. 

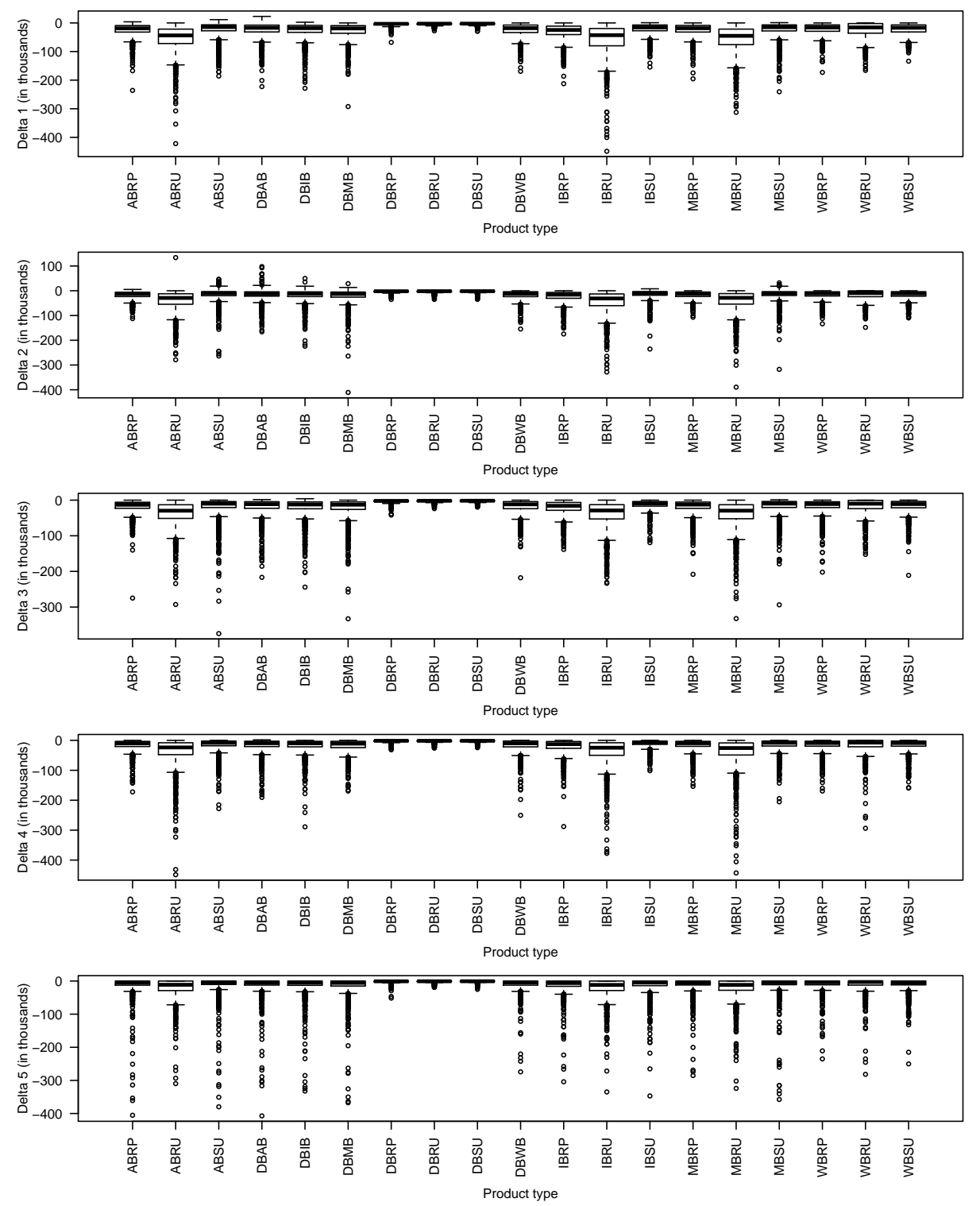

Figure 13: Box plots of the partial dollar deltas by product type at the end of year 1 of the worst real-world path. 


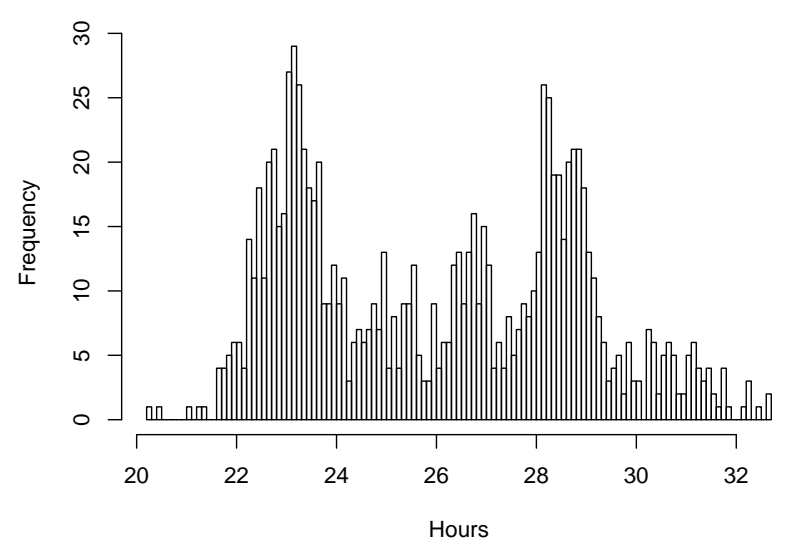

Figure 14: Distribution of the runtime for the 1,000 real-world paths.

\section{Acknowledgments}

This work is supported by a CAE (Centers of Actuarial Excellence) grant

2 from the Society of Actuaries.

Boyle, P. and Hardy, M. (1997). Reserving for maturity guarantees: Two approaches. Insurance: Mathematics and Economics, 21(2):113-127.

Carmona, R. and Durrelman, V. (2006). Generalizing the black-scholes formula to multivariate contingent claims. Journal of Computational Finance, $9(2): 43-67$.

Chopra, D., Erzan, O., de Gantes, G., Grepin, L., and Slawner, C. (2009). Responding to the variable annuity crisis. McKinsey Working Papers on Risk.

Dardis, T. (2016). Model efficiency in the U.S. life insurance industry. The Modeling Platform, (3):9-16.

Gan, G. (2013). Application of data clustering and machine learning in variable annuity valuation. Insurance: Mathematics and Economics, 53(3):795-801.

\footnotetext{
${ }^{2}$ http://actscidm.math.uconn. edu
} 
Gan, G. (2015). Application of metamodeling to the valuation of large variable annuity portfolios. In Proceedings of the Winter Simulation Conference, pages 1103-1114.

Gan, G. and Huang, J. (2017). A data mining framework for valuing large portfolios of variable annuities. In Proceedings of the 23rd ACM SIGKDD International Conference on Knowledge Discovery and Data Mining, pages $1467-1475$.

Gan, G. and Lin, X. S. (2015). Valuation of large variable annuity portfolios under nested simulation: A functional data approach. Insurance: Mathematics and Economics, 62:138 - 150.

Gan, G. and Lin, X. S. (2016). Efficient greek calculation of variable annuity portfolios for dynamic hedging: A two-level metamodeling approach. North American Actuarial Journal, In Press.

Gan, G., Ma, C., and Xie, H. (2014). Measure, Probability, and Mathematical Finance: A Problem-Oriented Approach. John Wiley \& Sons, Inc., Hoboken, NJ.

Gan, G. and Valdez, E. A. (2016). An empirical comparison of some experimental designs for the valuation of large variable annuity portfolios. Dependence Modeling, 4(1):382-400.

Gan, G. and Valdez, E. A. (2017). Valuation of large variable annuity portfolios: Monte carlo simulation and synthetic datasets. Dependence Modeling, $5: 354-374$.

Gan, G. and Valdez, E. A. (2018). Regression modeling for the valuation of large variable annuity portfolios. North American Actuarial Journal, $22(1): 40-54$.

Hardy, M. (2001). A regime-switching model of long-term stock returns. North American Actuarial Journal, 5(2):41-53.

Hejazi, S. A. and Jackson, K. R. (2016). A neural network approach to efficient valuation of large portfolios of variable annuities. Insurance: Mathematics and Economics, 70:169 - 181. 
Hejazi, S. A., Jackson, K. R., and Gan, G. (2017). A spatial interpolation framework for efficient valuation of large portfolios of variable annuities. Quantitative Finance and Economics, 1(2):125-144.

International Actuarial Association (2010). Stochastic Modeling: Theory and Reality from an Actuarial Perspective. International Actuarial Association, Ontario, Canada.

Ledlie, M. C., Corry, D. P., Finkelstein, G. S., Ritchie, A. J., Su, K., and Wilson, D. C. E. (2008). Variable annuities. British Actuarial Journal, 14(2):327-389.

The Geneva Association Report (2013). Variable annuities - an analysis of financial stability. Available online at: https://www.genevaassociation. org/media/618236/ga2013-variable_annuities.pdf.

Xu, W., Chen, Y., Coleman, C., and Coleman, T. F. (2018). Moment matching machine learning methods for risk management of large variable annuity portfolios. Journal of Economic Dynamics and Control, 87:1 - 20.

\section{Appendix A. Software and Datasets}

We implemented the nested stochastic valuation engine as a distributed multi-threading program in Java. The datasets and the software code can be downloaded from http://www. math.uconn. edu/ gan/software.html. 